\title{
Concept Validation for Selective Heating and Press Hardening of Automotive Safety Components with Tailored Properties
}

\author{
Nan Li ${ }^{1, a^{*}}$, Jianguo Lin ${ }^{1, b}$, Trevor A. Dean ${ }^{2, \mathrm{c}}$, Damian Dry ${ }^{3, \mathrm{~d}}$, Daniel Balint ${ }^{1, \mathrm{e}}$ \\ 'Department of Mechanical Engineering, Imperial College London, London, SW7 2AZ, UK \\ ${ }^{2}$ School of Mechanical Engineering, The University of Birmingham, Birmingham B15 2TT, UK \\ ${ }^{3}$ SAIC Motor UK Technical Centre Limited, Birmingham, B31 2BQ, UK \\ anan.li@imperial.ac.uk, ${ }^{b}$ jianguo.lin@imperial.ac.uk, ${ }^{c}$ t.a.dean@bham.ac.uk, \\ damian.dry@saicmotor.co.uk, ed.balint@imperial.ac.uk
}

Keywords: Hot stamping, boron steel, tailored properties, selective heating, press hardening

\begin{abstract}
A new strategy termed selective heating and press hardening, for hot stamping of boron steel parts with tailored properties is proposed in this paper. Feasibility studies were carried out through a specially designed experimental programme. The main aim was to validate the strategy and demonstrate its potential for structural optimisation. In the work, a lab-scale demonstrator part was designed, and relevant manufacturing and property-assessment processes were defined. A heating technique and selective-heating rigs were designed to enable certain microstructural distributions in blanks to be obtained. A hot stamping tool set was designed for forming and quenching the parts. Demonstrator parts of full martensite phase, full initial phase, and differentially graded microstructures have been formed with high dimensional quality. Hardness testing and three point bending tests were conducted to assess the microstructure distribution and load bearing performance of the as-formed parts, respectively. The feasibility of the concept has been validated by the testing results.
\end{abstract}

\section{Introduction}

The rising demand for increased safety and reduced weight of car bodies in the automotive industry has stimulated technological innovation in sheet metal forming [1]. Hot stamping, also called press hardening, of boron steel has been well developed. Now, considerable attention is being paid to improving the process, so as to produce parts with locally varying distributions of mechanical properties, which introduces the potential for making parts conforming more fully to functional requirements [2]. Comprehensive studies have been carried out to tune microstructure distributions by selectively cooling the part, which is called the tailored tempering process, or partial press hardening [3]. However, because a long cooling time is required to obtain ductile phases, there is an intrinsic conflict with the industrial requirement for short cycle times [4]. Therefore, a novel concept is proposed in this study for optimising the structural performance of a press-hardened component by means of austenite transformation control through differential heat treatment of the blank prior to forming. This process strategy is called selective heating and press hardening in this paper [5].

Concept illustration. The concept in the study is the optimisation of structural performance of press-hardened parts by means of microstructural design, realised by controlling thermal conditions during preheating in the hot stamping processes. Fig. 1 schematically shows in simplified form the distribution of microstructure in the work-piece and formed part, and the method of assessing mechanical properties. Fig. 1 (a) illustrates a portion S/L of a work-piece, the hot zone, fully austenitized by heating to and soaking at a temperature of $1173 \mathrm{~K}$. The two ends, the warm zones, are heated to a temperature below the austenitization start temperature, 993K [6]. The warm zones are composed of the initial phase, and are multiphase in the transition regions adjacent to the hot 
zone. Fig. 1 (b) shows the pressed shape, representing an impact beam, which has been die quenched and is fully martensitic in the prior hot zone. Fig. 1 (c) shows load bearing assessment of the as-formed part through three point bending test. The performance of the part would vary with the length of the full martensite zone (hard zone), and this can be optimised in the selective heating operation to comply with a particular design specification.

(a)

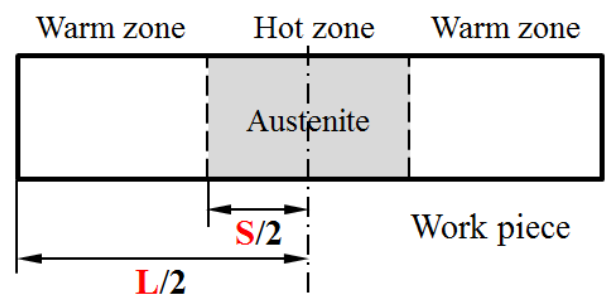

(b)

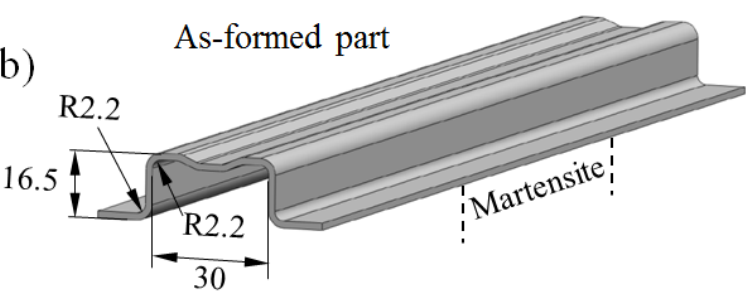

(c)

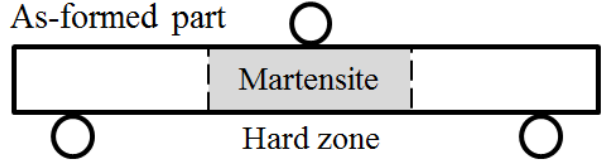

Fig. 1 Schematics illustrating the manufacturing process and experimentation for function assessment: (a) Selective heating of the work-piece (b) An as-formed demonstrator part after hot stamping and cold die quenching, with the dimensions (mm) of the cross-section (c) Three point bending of the as-formed part.

\section{Experimental design}

Material and demonstrator part. The material used in this study was a 22MnB5 manganese-boron steel provided by the project sponsor SAIC MOTOR. It was cold rolled and hot-dip aluminized. The following product information was provided by steel supplier: the initial microstructure contained a 78/22 mixture of proeutectoid ferrite and pearlite; the chemical composition of the investigated steel is listed in Table 1. All work-pieces used for the experiment were machined from the same batch of as-delivered, $1.6 \mathrm{~mm}$ thick boron steel sheet.

Table 1. Chemical composition (Max value - ladle analysis in \%).

\begin{tabular}{cccccccc}
\hline $\mathrm{C}$ & $\mathrm{Si}$ & $\mathrm{Mn}$ & $\mathrm{P}$ & $\mathrm{S}$ & $\mathrm{Cr}+\mathrm{Mo}$ & $\mathrm{Ti}$ & $B$ \\
\hline 0.25 & 0.40 & 1.40 & 0.025 & 0.010 & 0.50 & 0.05 & 0.005 \\
\hline
\end{tabular}

Fig. 1 (b) shows the cross-section of a lab-scale demonstrator part, the length (L) of which was 320 $\mathrm{mm}$. The dimensions of the initial blank were $80 \mathrm{~mm} \times 320 \mathrm{~mm}$.

Test programme. By taking the length of the hot zone, $S$, as a variable, four test conditions were defined and they are listed in Table 2, where S-60 mm and S-100mm are two conditions designed for selective heating with $60 \mathrm{~mm}$ and $100 \mathrm{~mm}$ hot zones, respectively, and S- $0 \mathrm{~mm}$ and S-320mm represent uniform heating without a hot zone and with a full hot zone, respectively. In addition, assessment of both microstructural and structural properties of as-formed parts was conducted for conditions presented in Table 2.

Table 2. Test programme (' $\sqrt{ }$ ' represents the selected test conditions).

\begin{tabular}{|c|c|c|c|c|}
\hline \multirow{2}{*}{$\begin{array}{c}\text { Test } \\
\text { condition }\end{array}$} & \multirow{2}{*}{$\begin{array}{c}\text { Hot } \\
\text { forming }\end{array}$} & \multicolumn{2}{|c|}{ Hardness distribution } & \multirow{2}{*}{ Bending tests } \\
\hline & & Sectional & Longitudinal & \\
\hline $\mathrm{S}-0 \mathrm{~mm}$ & $\sqrt{ }$ & & & $\sqrt{ }$ \\
\hline $\mathrm{S}-60 \mathrm{~mm}$ & $\sqrt{ }$ & & $\sqrt{ }$ & $\sqrt{ }$ \\
\hline $\mathrm{S}-100 \mathrm{~mm}$ & $\sqrt{ }$ & & $\sqrt{ }$ & $\sqrt{ }$ \\
\hline $\mathrm{S}-320 \mathrm{~mm}$ & $\sqrt{ }$ & $\sqrt{ }$ & & $\sqrt{ }$ \\
\hline
\end{tabular}




\section{Heating technique}

Selective heating is an essential part of the strategy. In this work, it was realised by local contact heat treatment in a furnace under laboratory conditions. The implementation is shown in Fig. 2 (a) and (b). A selective heating rig, consisting of two heating blocks made of stainless steel, served as an energy reservoir for locally heating a blank in a hot zone. It was positioned in a furnace and preheated to a temperature above $1173 \mathrm{~K}$. Heat transfer in the hot zone took place when a cold workpiece was positioned between two hot blocks. At the same time, the two ends of the work-piece, the warm zones, mainly heated by radiation and convection within the furnace, were thus heated at a lower rate and their maximum temperature was controlled to just below $993 \mathrm{~K}$. With respect to the selective heating rig, in Fig. 2 (b), the blocks were massive to ensure sufficient heat capacity. In addition, the top block was designed to be larger to provide higher pressure between the work-piece and blocks and enhance heat transfer. Ceramic plates were inserted between the blocks to give them a $1 \mathrm{~mm}$ gap during preheating, in order to enable easy separation before positioning the work-piece. Two pairs of hot blocks were made for $60 \mathrm{~mm}$ and $100 \mathrm{~mm}$ hot zones. To enable the two selective heating conditions, S-60mm and S-100mm, to share a unified process control, the heating rig should be of the same heat capacity for both conditions. Since hot blocks for S-100s were wider, an additional block was placed beneath the selective heating rig for the S-60mm condition, so that the total volume of the heating blocks was the same as that for the $\mathrm{S}-100 \mathrm{~mm}$ case.

Effective control of thermal conditions to ensure a desired temperature distribution in a work-piece was required. Therefore, thermocouples were attached to a work-piece to monitor temperature profiles and enabled a suitable heating procedure to be determined. Fig. 2 (c) shows the location of thermocouples on the work-piece. In order to avoid possible influence on the contact between the work-piece and blocks, small holes were drilled perpendicular to the through-thickness section to fix K-type thermocouples of $0.5 \mathrm{~mm}$ in diameter. The output ends of the thermocouples were connected to a National Instruments data logger for data acquisition, as shown in Fig. 2 (a).

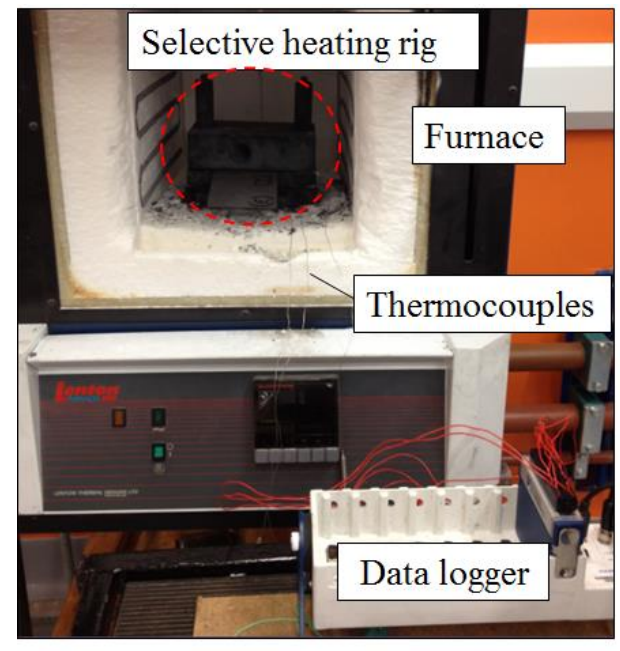

(a)

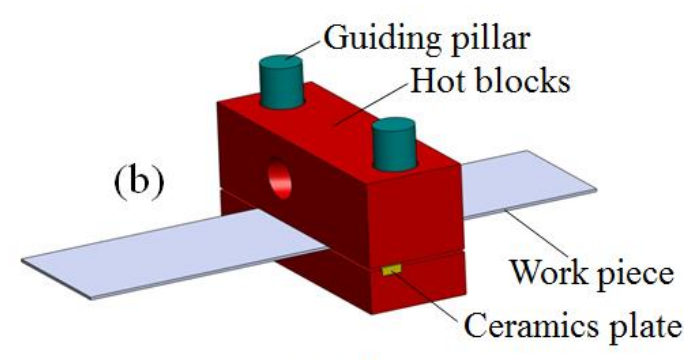

S-60mm

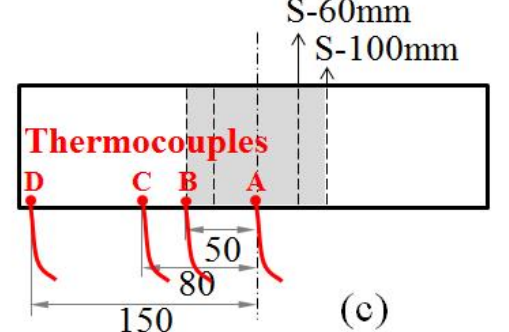

Fig. 2 Selective heating technique (a) Set-up of facilities for selective heating and temperature measurement (b) 3-D diagram of the selective heating rig (c) Location of thermocouples attached to a work-piece (dimensions in the figure are in $\mathrm{mm}$ ).

For uniform heating, S-0mm and S-320mm, no hot blocks were used and the work-piece was put into the furnace with environmental temperatures of $973 \mathrm{~K}$ and $1173 \mathrm{~K}$, respectively, for 500 seconds. For selective heating conditions, since the preheated rig was a massive heat resource in the small chamber, the reduction of environmental temperatures had to be realised by opening the chamber at intervals to enhance air convection. The temperature profiles were decided by the preheating temperature of the hot blocks and the time to increase and reduce air convection. Trial 
and error was adopted, aiming to achieve full austenitization in the hot zone with no phase transformation in the warm zones. Fig. 3 shows the summary of final temperature profiles obtained for the four test conditions. Representative positions are chosen, for example, ' $\mathrm{S}-100 \mathrm{~mm}-\mathrm{A}$ ' represents the hot zone temperature measured by thermocouple $\mathrm{A}$ for the $\mathrm{S}-100 \mathrm{~mm}$ test condition. Good consistency for S-60mm and S-100mm was achieved. As seen in the figure, full austenitization was expected at the hot zone for selective heating conditions, same as that for the uniform heating condition S-320mm; no phase transformation was expected at the portion D of the warm zones, same as that for $\mathrm{S}-0 \mathrm{~mm}$. The adjustment of air convection is revealed by the sawtoothshape of the temperature profiles for the warm zones ('S-100mm-D' and ' S-60mm-D'). The amplitude of these temperature fluctuations can be reduced by increasing the frequency and decreasing the time intervals of air convection. However, the feasibility of manual controlling the procedure limited the rate at which the operations could be undertaken.

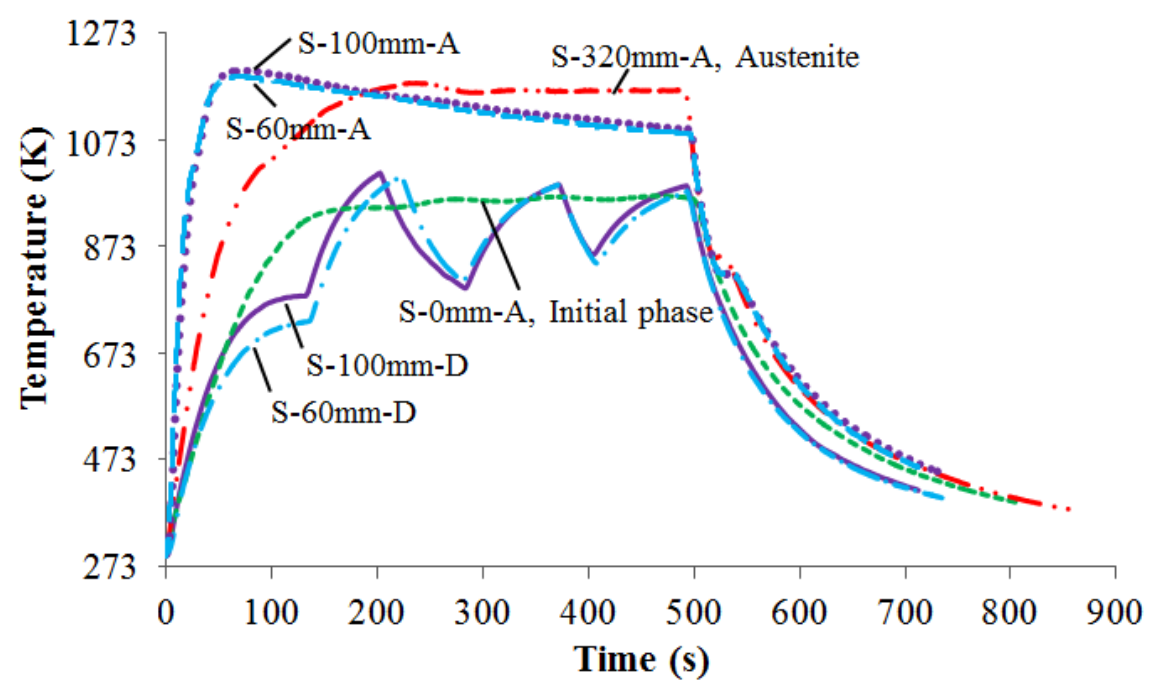

Fig. 3 Temperature profiles for 4 different test conditions.

In addition, from Fig. 3, if the time of transfer of the work-piece from the furnace to the tools after soaking is known, the natural cooling curves can be used to estimate the starting forming temperature for various conditions. The determined thermal parameters and operation procedure for selective heating were used as standards for all following hot stamping tests.

\section{Hot stamping}

Design of hot stamping tool set. A hot stamping tool set was designed to be set on a hydraulic press for forming the demonstrator part. The two main functions of the tool are to form the workpiece and extract heat from it at a high rate [7], thus the design work was associated with careful consideration of both material flow and heat transfer.

The tool was designed to enable a part with as uniform a thickness as possible to be produced. A section of the tool is presented in Fig. 4 to schematically illustrate its working mechanism. To properly arrange the material flow, the top die was designed to be composed of two parts. The main part of the top die (sides in Fig. 4) was fixed to the ram of the press and the punch was seated on gas springs to allow relative movement. Fig. 4 (a) shows the start of forming when the work-piece was in contact with the punch. With the whole top die set moving down, the middle groove was formed and, as shown in Fig. 4 (b), the punch was brought to rest by the counterpunch while the sides continued to move downwards until the part was fully formed, as shown in Fig. 4 (c). Arrows in solid lines represent forming occurring under the particular tool part. Little stretching of the blank occurred and it was subjected to near pure bending. Since heat transfer coefficient depends on contact pressure, tight contact between work-piece and tool is essential. In this design, see Fig. 4 (c), 
the flexible top die set guaranteed tight contact over the interfaces (1), (2), and (3). In addition, the two side parts of the bottom die were also adjustable, which ensured tight contact over the interfaces (4) and (5). An additional feature of the tool was that beams with different groove depths could be formed by changing the thickness of a replaceable block in the counterpunch. For 22MnB5 manganese-boron steel, the minimum value of quenching rate required to achieve full martensite is for the undeformed state, and is $27 \mathrm{~K} / \mathrm{s}$ [8]. Since the tool set was for laboratory applications instead of mass production, no water cooling channels were needed in the dies. The dies were made of AISI type H13 hot work tool steel. The die clearance was $0.1 \mathrm{~mm}(6.25 \%)$ for the $1.6 \mathrm{~mm}$ thick workpiece.

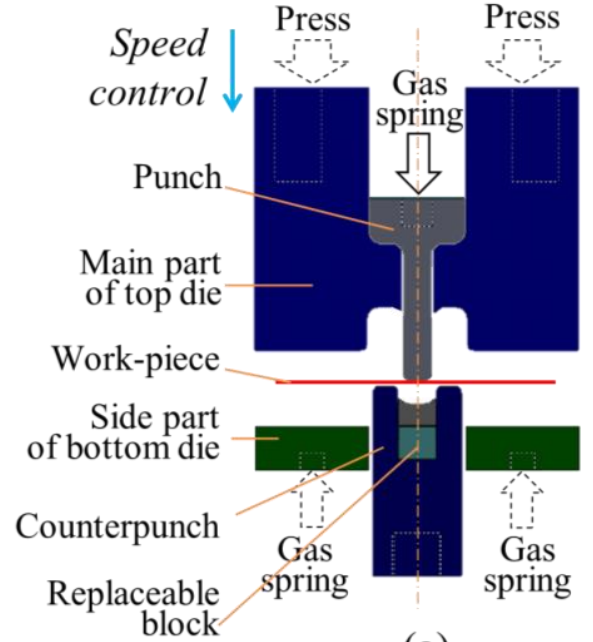

(a)

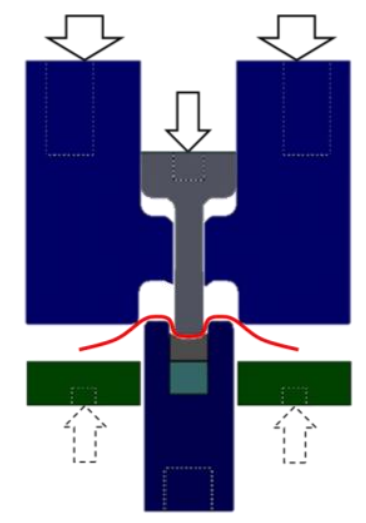

(b)

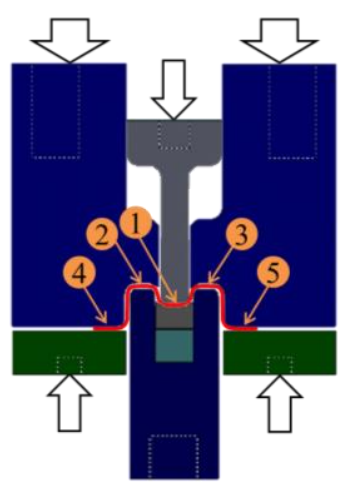

(c)

Fig. 4 Schematics illustrating the working mechanism of the tool set and material flow of the work-piece (a) Start of forming (b) during forming, middle groove has been formed (c) final stage of forming.

Hot stamping experiments. Hot stamping experiments were carried out on a $1000 \mathrm{kN}$ hydraulic testing machine. The set-up of facilities is shown in Fig. 5. The furnace was used for heat treatment of work-pieces, determined as described in the previous section. The controller was used to operate the machine. A load cell was set on top of the tool set to measure the forming force. The forming speed was $150 \mathrm{~mm} / \mathrm{s}$, which was determined to be appropriate to industrial processes, giving an average strain rate of $0.5 / \mathrm{s}$, approximately. Forming force and punch displacement were monitored through a digital work station, to ensure that the load provided by the machine was sufficient and the forming speed was accurate. The time for transferring blanks to the tool set was controlled at approximately 3 seconds. The times for positioning and forming were about 1 and 0.2 seconds, respectively. Formed parts were held in the dies for about 20 seconds.
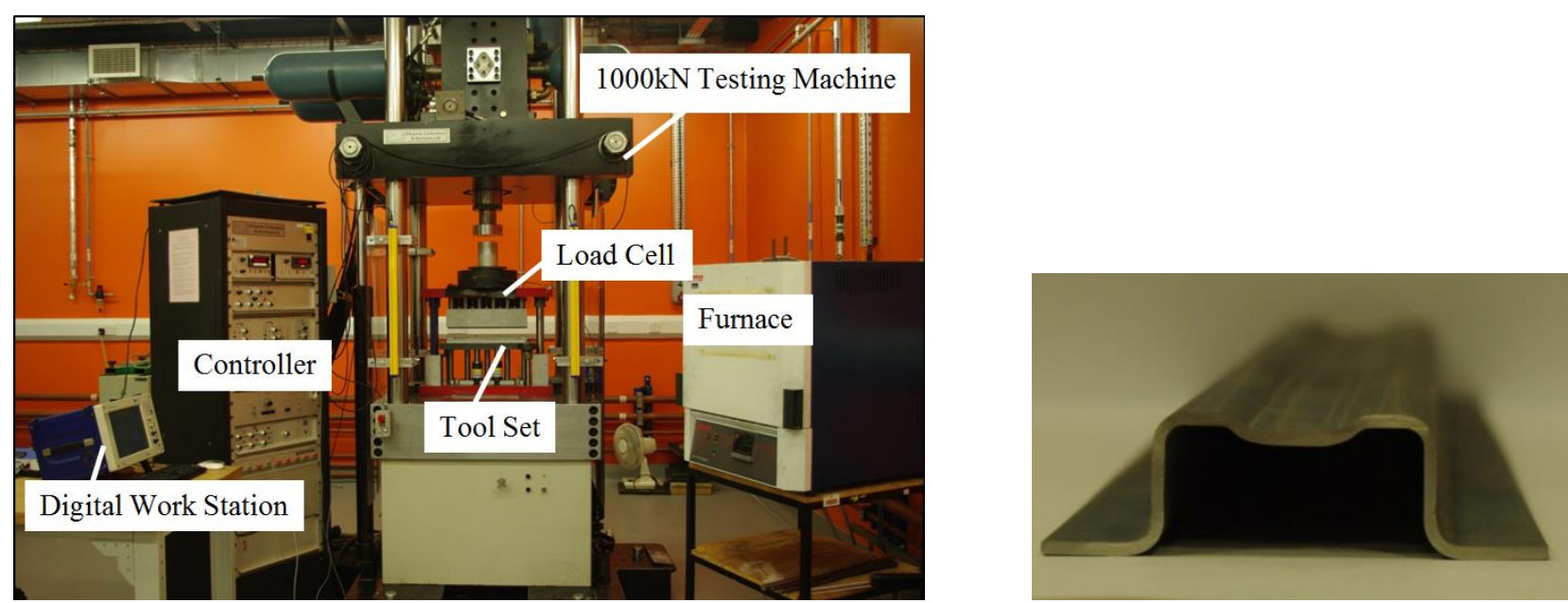

Fig. 5 Set-up of facilities for hot stamping experiment. Fig. 6 Cross-section view of a formed part. 
Fig. 6 shows the cross-section of a formed product. Formed products with different microstructural topographies all exhibited good quality without visible spring-back. This suggests that, even without a phase transformation, the residual stress in the part could be eliminated by holding and quenching within closed cold dies.

\section{Property assessment}

Hardness testing. Hardness testing was conducted on as-formed parts to confirm complete martensitic transformation had been achieved through the cross-section of the prior hot zone, and to detect the microstructural distribution along the length of parts that were selectively heat treated. The Vickers hardness values of the studied boron steel are $\geq 475 \mathrm{HV}$ for martensite and $160 \mathrm{HV}$ for as-delivered initial phase [6].

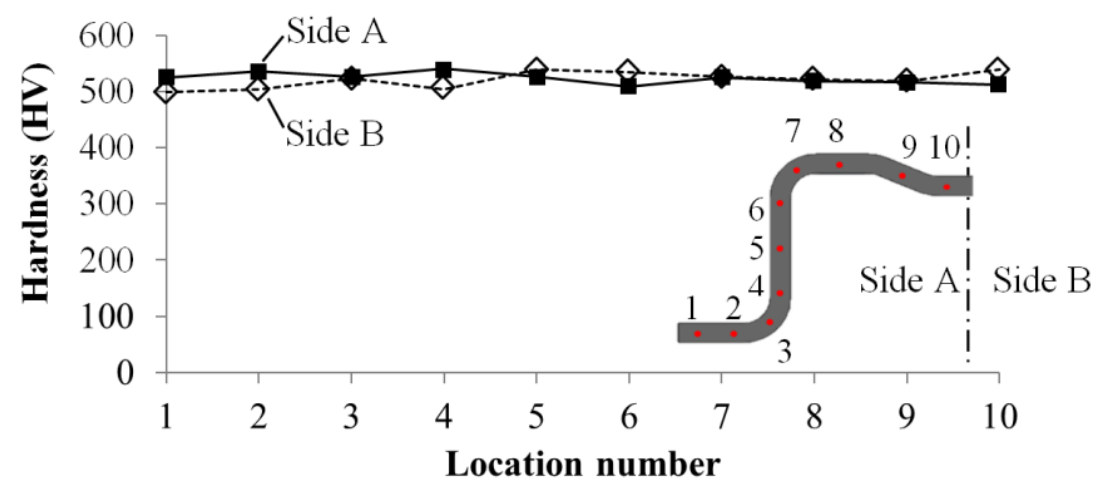

(a)

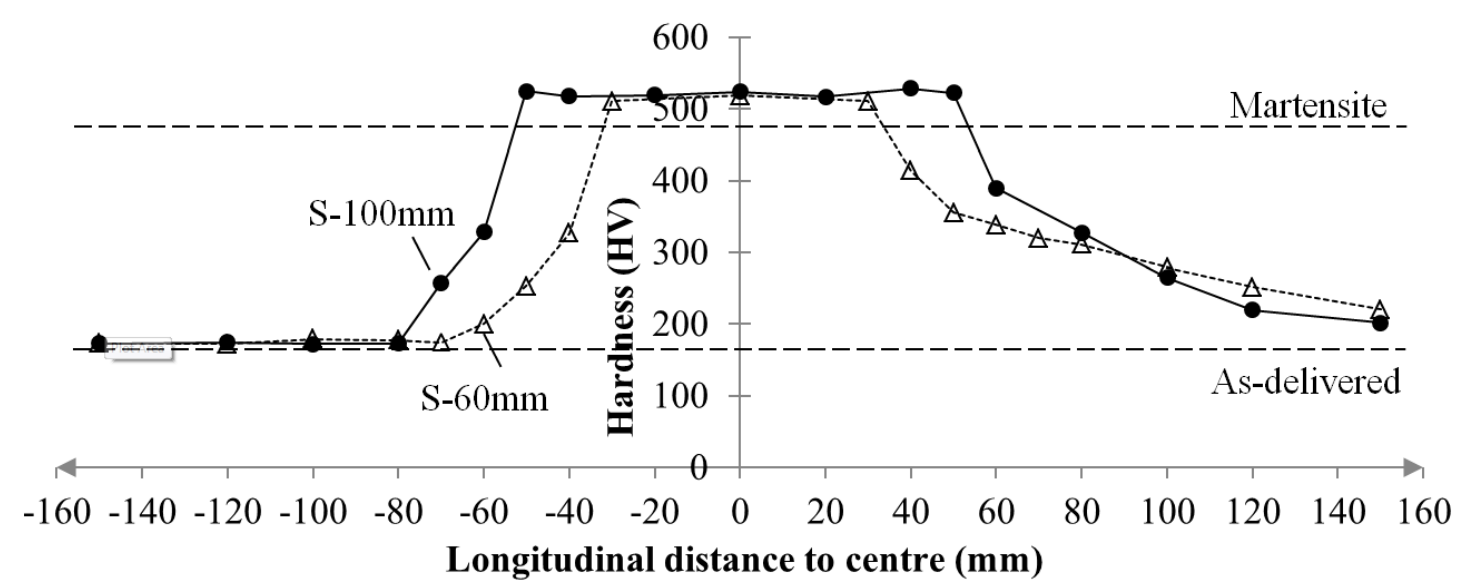

(b)

Fig. 7 Hardness distributions (a) through a cross-section of an S-320mm as-formed part (b) along the longitudinal direction of S-60 $\mathrm{mm}$ and S-100mm as-formed parts.

In Fig. 7 (a), hardness values from twenty positions through the cross-section of an S-320mm formed part are plotted. The numbering of locations for indentation on the half section (Side A) is shown in the figure. Due to the section being symmetrical, the numbering system is mirrored on side B. It can be seen that all hardness values are $520 \pm 20 \mathrm{HV}$, indicating a full martensite distribution along the section. This implies good contact between the hot part and tool. In Fig. 7 (b), hardness values along the longitudinal direction of S-60mm and S-100mm as-formed parts are plotted on a length scale, where the beam mid-length is taken as the origin. Indentations were applied along the lengths of the top surfaces of flanges, $1.5 \mathrm{~mm}$ from the edges, of the parts. For both the parts, full martensite was achieved in the prior hot zones with hardness values of $520 \pm 10 \mathrm{HV}$, which suggests full austenitization has been achieved in pre-heating. Away from the hot zone at both sides, the inter-zone regions with gradually decreasing hardness values are transition zones having graded multiphase microstructures. The region with almost constant hardness of $175 \pm 5 \mathrm{HV}$ at the left end 
indicates that the initial phase was retained without phase transformation. Hardness values on the right side show a slightly higher level for each part. This was due to a higher temperature at the back of the heating blocks in the furnace resulting from reduced air convection.

Microstructural distribution varies with many factors consisting of both material thermal properties and thermal conditions, such as thermal conductivity of the alloy, distribution of heating temperature, and soaking time. Besides control of the martensite and initial phase, there is considerable room to grade the microstructures in the transition zones. For example, in this work, the gradient of hardness corresponding to warm zones was affected by air convection.

Three point bending tests. To assess the load bearing performance of the as-formed parts, three point bending tests were conducted on an INSTRON $(5585 \mathrm{H}) 250 \mathrm{KN}$ universal test machine. The flex fixture can be seen in Fig. 8 . The beams were $320 \mathrm{~mm}$ long, loaded in the middle by an indenter with $40 \mathrm{~mm}$ diameter. The indenter speed was $0.5 \mathrm{~mm} / \mathrm{s}$. Fig. 8 shows the failure modes of representative as-formed boron steel beams with different microstructural topographies. For beams with a uniform microstructure, failure took place at the indenter, where the bending moment was maximum. For beams with graded microstructures, the local instability initiated at a position away from the hard (martensite) zone, in a region associated with mixed microstructural phases and therefore a range of mechanical properties. This suggests that the deformation mode of a part can be adjusted through microstructural arrangement, for specific functional requirements.
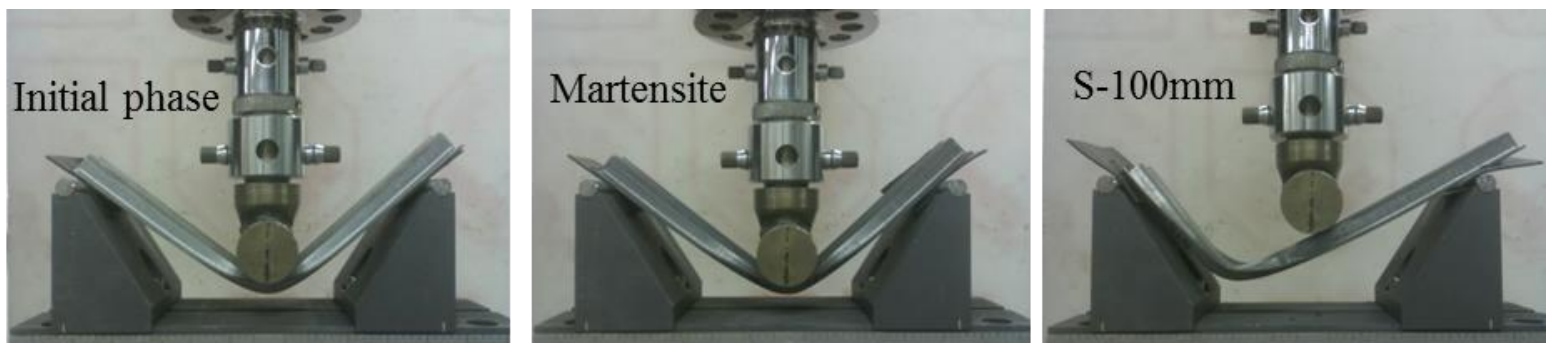

Fig. 8 Failure modes under bending for boron steel parts having different microstructural topographies.

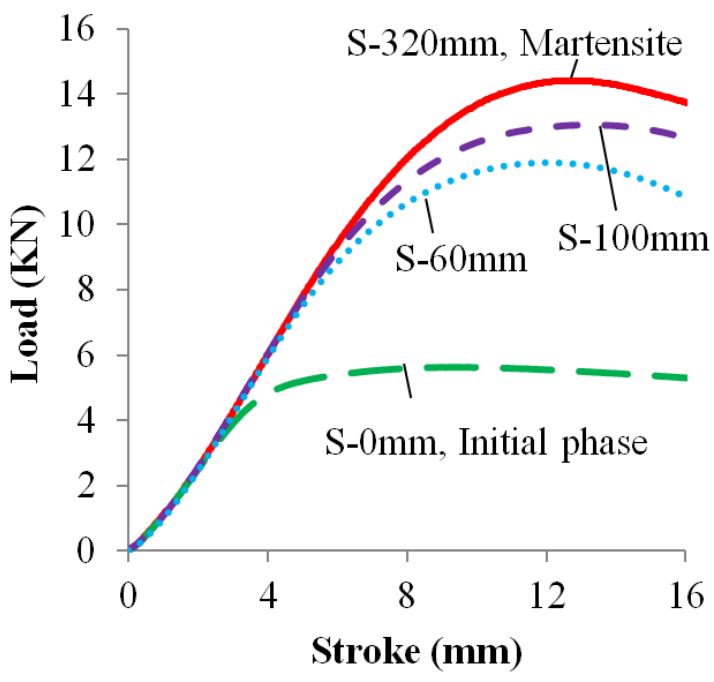

(a)

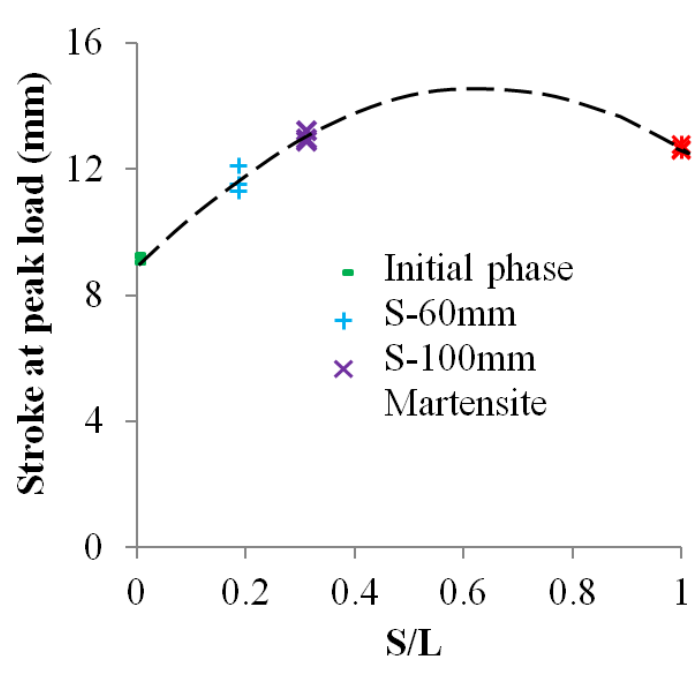

(b)

Fig. 9 Load bearing performance of boron steel parts having different microstructural topographies: (a) Load deflection curve (b) Stroke at the onset of instability against the proportional length of full martensite phase. 
Fig. 9 (a) shows the load deflection curves of the beams corresponding to the four microstructural topographies. The fully hardened beam $(\mathrm{S}-320 \mathrm{~mm})$ sustained the highest load which was about three times that for the beam with initial phase $(\mathrm{S}-0 \mathrm{~mm})$. The beam $\mathrm{S}-100 \mathrm{~mm}$ endured a lower load than beam S-320mm but deformed more before the onset of instability. This behaviour can be attributed to the existence of advantageous portions of strong and ductile regions in the beam. Fig. 9 (b) shows the relation between degree of stable deformation and length of full martensite phase. Three data points are plotted for each condition. From the curve it can be inferred that the greatest energy absorption (high load and large stable deformation) is obtained for an S/L value of between 0.6 and 0.7. In addition, the performance of a beam with tailored properties might be improved if it contained a more symmetrical microstructural distribution and a better graded multiphase zone.

\section{Conclusions}

The selective heating and press hardening strategy has been successfully implemented in a hot stamping operation. Demonstrator parts, with different microstructural topographies, have been formed with good quality and without visible spring-back. Microstructural distributions in asformed parts were revealed by hardness testing. The structural performance of as-formed parts under three point bending has been determined. Based on experimental results, the following conclusions have been drawn:

- Differentially heated blanks can be press hardened with high shape quality, which is essential for commercial viability of the forming process.

- Microstructural distribution in formed parts can be graded by the design of thermal conditions, which provides the basis for optimising structural properties of a part.

- The deformation mode of a part can be adjusted through microstructural arrangement; the energy absorption capacity of a part can be improved through advantageous combination of strong and ductile regions. Thereby, the potential of the selective heating and press hardening strategy in functional optimisation is demonstrated.

\section{Acknowledgements}

The authors thank SAIC Motor UK Technical Centre for financial support.

\section{References}

[1] H. Karbasian. and A.E. Tekkaya, A review on hot stamping, Journal of Materials Processing Technology, 2010. 210(15): p. 2103-2118.

[2] N. Li, et al. Investigation on the mechanical properties of as-formed boron steels for optimizing process strategies in hot stamping, the 14th International Conference of Metal Forming, 2012.

[3] D. Thomas and D.T. Detwiler, Microstructural optimization of automotive structures, HONDA MOTOR CO., LTD. (Tokyo, JP), U.S. Patent 20090242086, 2009

[4] N. Li, et al., Experimental characterisation of the effects of thermal conditions on austenite formation for hot stamping of boron steel, submitted to Philosophical Magazine, 2014.

[5] N. Li, Fundamentals of materials modelling for hot stamping of UHSS panels with graded properties, $\mathrm{PhD}$ thesis, Department of Mechanical Engineering, Imperial College London, 2013

[6] ThyssenKrupp Steel, Hot press hardening manganese-boron steels MBW for ultrahigh strengths, 2008.

[7] A. Naganathan and L. Penter, Sheet metal forming - processes and applications, Hot stamping, ASM International, 2012.

[8] J. Cai, Modelling of phase transformation in hot stamping of boron steel, $\mathrm{PhD}$ thesis, Department of Mechanical Engineering, Imperial College London, 2011 